\title{
ELLIS-VAN CREVELD SYNDROME
}

\author{
Yaşar Cesur ${ }^{1}$, Sevil Arı Yuca ${ }^{1}$, Abdurahman Üner ${ }^{2}$, Köksal Yuca ${ }^{3}$, Derya Arslan ${ }^{4}$
}

Yüzüncü Yıl University, Fakulty of Medicine, Department of Pediatric Endocrinelogy', Pediatric Kardiyology ${ }^{2}$, Otolaryngology ${ }^{3}$, and Pediatrics ${ }^{4}$, Van, Turkey

Ellis-van Creveld syndrome (EVCS) or chondroectodermal dysplasia is a rare autosomal recessive disorder characterized by a variable spectrum of clinical findings. Classical EVC syndrome comprises a tetrad of clinical manifestations of chondrodystrophy, polydactyly, ectodermal dysplasia, and cardiac defects.

The patient presented with short stature, polydactyly, triangular face and dental dismorphism was found appropriately as radiographic with ectodermal dysplasia. In addition to the presence of single atrium, mitral, tricuspid and pulmonary valve insufficiency and pulmonary hypertension wererevealed by echocardiography. Her history involved a sister who was dead in 3-month-ages.

Key words: Ellis-van Creveld (EVC) syndrome, chondroectodermal dysplasia, single atrium, pulmonary hypertension.

Eur J Gen Med 2008; 5(3):187-190

\section{INTRODUCTION}

Ellis-van Creveld syndrome (EVCS) is a complex genetic syndrome first described in 1940 by Drs Richard Ellis and Simon van Creveld. Classical EVCS comprises a tetrad of clinical manifestations, chondrodysplasia, ectodermal dysplasia, polydactyly and congenital heart disease (1). Although this tetrad constitutes the classical syndrome description, a variable spectrum of clinical manifestations is frequently present. Other organs of endodermal origin are sometimes affected in EVCS. Pulmonary, renal, hepatic, pancreatic, and central nervous system abnormalities have been reported previously but constitute some of the rarer syndrome associations (2).

We present the case of a 14-year-old girl with EVCS and characteristic findings, including narrow thorax, short limbs, bilateral polydactyly of the hands and foots, short-limb dwarfism, hypoplastic spoon-shaped nails.

\section{CASE}

The patient was brought to our clinic because of short arms and legs, polydactly and partially absent of teeth. She was born at 39 weeks gestation and was the product of consanguineous marriage and normally developed parents. A pregnancy was uneventful but the patient was born with small for gestational age. Antenatal, natal and neonatal histories were noncontributory. There was a family history of neonatal death of a male and two female siblings due to unclear etiology. While a female sibling had polydactyl her other three siblings with healthy.

The patient's physical findings included short-limb dwarfism (her height is 126 $\mathrm{cm},-5.6 \mathrm{SDS}$ ), hypoplastic spoon-shaped nails, low set shoulders, narrow chest, genu valgum and bilateral polydactyly of the hands and foots. His facial and dental anomalies included gum abnormalities and supernumerary upper teeth including one conical in shape. Cardiovascular examination revealed a systolic murmur at the left parasternal area and mitral area. Her psychomotor and mental developments were retarded. Radiographic surveys revealed distal ward shortening of the tubular bones, bilaterally polydactyly of the hand and foot, carpometacarpal fusion

Correspondence: Sevil Arl Yuca MD.

Department of Pediatrics, Yuzuncu Yil University, Faculty of Medicine, Van, Turkey.

Tlf: 0904322140501, Fax:0904322140501

E-mail:sevilyuca@yahoo.com 


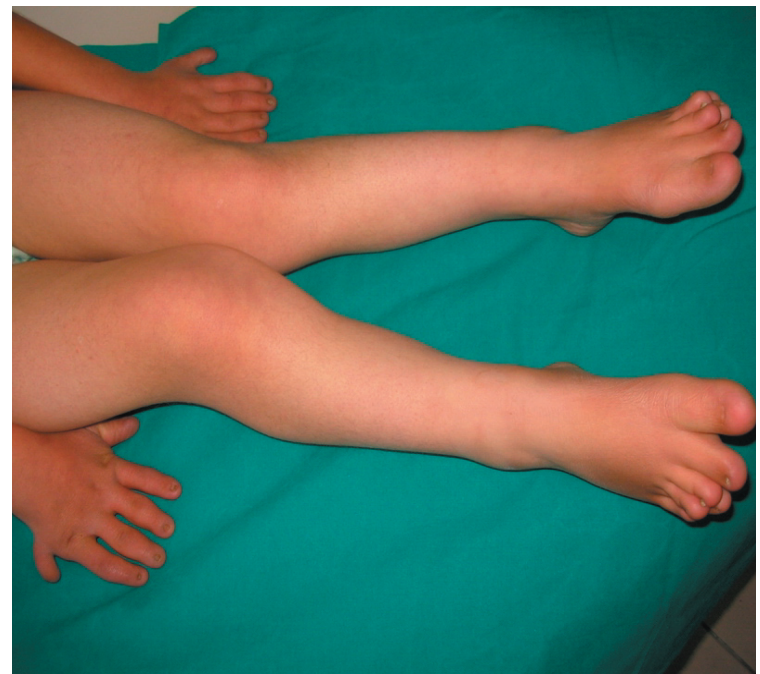

Figure 2. A patient with EVCS shows genu valgum, polidactyly and hyphoplastic fingernails and toenails.

of the left hand and partial fusion of the fifth and sixth metatarsals, clinodactyly, shortening phalanx of the hand and foot, genu valgum. In echocardiographic examination; it had been found a single atrium, mitral, tricuspid and pulmonary valve insufficiency and pulmonary hypertension. A clinical diagnosis of EVC syndrome was made based on the physical and radiographic findings which are strongly characteristic for chondroectodermal dysplasia.

\section{DISCUSSION}

EVCS is an autosomal recessive skeletal dysplasia. Although is very common in one Amish group (3) it does not show racial predilection and it does not show gender predilection $(5,6)$. The risk of recurrence for siblings is 1 in 4 (25\%) for each offspring. For children of an affected person the risk is not increased, unless the spouse is also a carrier, in which event the risk is $50 \%$ (6). Goldblatt et al. described EVC in a Western Australian Aboriginal community in which 2 relatives were observed to have isolated postaxial polydactyly of the feet. They advanced this as evidence of heterozygous manifestation (6). A linkage analysis of several families of different ethnic origin revealed linkage to 4 p16.1 (7). Ruiz-Perez et al. 2000 described mutations in EVC in patients with this condition and later identified different novel genes (8-10). Isolated findings in near relatives, such as polydactyly, short stature or abnormalities of wrist bones without other stigmata of the syndrome, have been frequently described (11). Our patient is female and there was a family history of neonatal death of a female sibling who has polydactyl. Her parents have been consanguineous marriage but they haven't similar diseases.

The condition is a type of dwarfism characterized by multiple anomalies of the mesodermal and ectodermal tissue. The cardinal symptoms are as follows: bilateral postaxial polydactlyly of the hands, ectodermal dysplasia (multiple frenula, dysplastic and hypoplastic nails), chondrodysplasia resulting in shortness of the long bones (predominantly of the mesomelic bones), and congenital heart defects $(1,3,8)$. There was short stature, short arms, narrow chest, polidactily, expended methacarps-methatarses in hands and foods, and hyphoplastic fingernails and toenails in our case (figure 1-2).

In EVCS, abnormalities of the oral cavity are common. These include frenulae or areas of fusion between the inner upper lip and gum, and partial or pseudo clefts of the upper lip. Teeth problems include teeth present at birth (natal teeth), missing teeth or peg-shaped teeth. Although case reports of EVCS are scarce, a thorough review found of airway anomalies $(11,12)$. Our patient ear-nose -throat examinations were included high plate, hypertrophy of palatine and adenoid tonsils, missing peg-shaped teeth (figure 3 ). She had distressed of breath but we found airway anomalies teeth and, we think that hypertrophy of adenoid tissue might have causation this condition.

The essential radiological findings include progressively in distal ward shortening of segments and generalized thickness of bones. Radiological features such as curvature of the humerus, enlargement of distal ends of radii and ulnae, supernumerary carpal bone centers with fusion, clinodactyly, synmetacarpalism, synostosis, wedgeshaped tibia epiphyses and genu valgum vary depending on disease severity and age.

Classical chondrodystrophic changes involving the tubular bones were observed on radiological examination in our case that is in accordance with previous reports. 


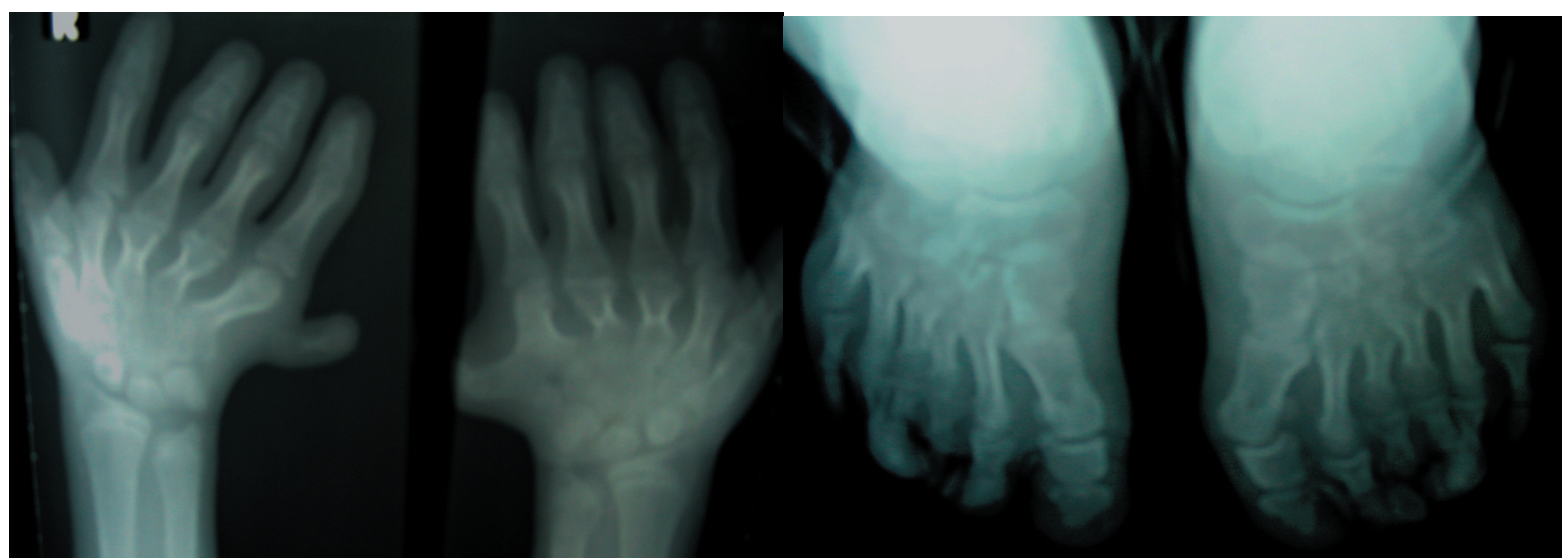

Figure 4. A patient's hand and wrist graphics with EVCS shows carpometacarpal fusion in left hand and, partial fusion in between five and six metacarpals and polydactyly in both of hands.

There were findings as accordance with syndrome in our patient's bone graphics which were polydactyly of the both hands and feet, carpometacarpal fusion in the left hand and, partial fusion of the five and six metacarpals and metatarses of both hands and feet, genu valgum and hypoplasia of the costales (figure 4-5).

In addition to the characteristic triad of EVCS, the heart defects are the most common abnormalities. Congenital cardiac defects, most commonly a defect of primary atrial septation producing a common atrium, occur in $60 \%$ of affected individuals. Other abnormalities may accompany insufficiency these lesions, such as aortic atresia, hypoplasia of the ascending aorta or of the left ventricle. cleft mitral valve, atrial septal defect, ventricular septal defect, transposition of big arteria, mitral valve, double orifice mitral valve, cleft mitral valve (13-15). The patient's ecocardiographic findings were single atrium, mitral, tricuspid and pulmonary insufficiency and pulmonary hypertension.

Although, most of the patients with EVC have normal intelligence, mental retardation and central nervous system abnormalities were reported in some cases $(16,17)$. Also genito-urinary system abnormalities may be associated with this syndrome (16). The patient who was mentioned in here, didn't have any abnormality of urinary and central nervous system.
Figure 5. A patient's feet graphics shows partial fusion of the five and six metatarses and polydactyly of feet.
In conclusion, we pointed that EVCS might be remembered when a patient has skeletal dysplasia characterized by short limbs, short ribs, short stature, polydactyly, and dysplastic nails and teeth. It also possible to prenatal diagnose with ultrasonography in uteri for siblings with affected person (18-20). The early diagnosis will be feasible in following pregnancies by well recognizing of clinical findings of this syndrome.

\section{REFERENCES}

1. Ellis RWB, Van Creveld SA. Syndrome characterized by ectodermal dysplasia, polydactyly, chondro-dysplasia and congenital morbus cordis: report of three cases. Arch Dis Child 1940;15:65-84

2. Scurlock D, Ostler D, Nguyen A, Wahed A. Ellis-van Creveld Syndrome and Dyserythropoiesis Arch Pathol Lab Med 2005;129:680-2

3. McKusick VA; Egeland JA; Eldridge R, Krusen DE. Dwarfism in the Amish. I. The Ellis-van Creveld syndrome. Bull Johns Hopkins Hosp 1964;115:306-36

4. Oliveira da Silva $E$, Janovitz $D$, de Alberquerque SC. Ellis-van Creveld syndrome: Report of 15 cases in an inbred kindred. J Med Genet 1980;17:349-56

5. Goldblatt J, Minutillo C, Pemberton PJ, Hurst J. Ellis-van Creveld syndrome in a Western Australian Aboriginal community: postaxial polydactyly as a heterozygous manifestation?. Med J Aust 1992;157:271-2

6. Varela M, Ramos C. Chondroectodermal 
dysplasia (Ellis-van Creveld syndrome): a case report. Eur J Orthod 1996;18:313-8

7. Polymeropoulos, MH, Ide SE, Wright M, Goodship J, Weissenbach J et al. The gene for the Ellis-van Creveld syndrome is located on chromosome 4p16. Genomics 1996;35:1-5

8. Ruiz-Perez VL, Ide SE, Strom TM, Lorenz $B$, Wilson $D$ et al. Mutations in a new gene in Ellis-van Creveld syndrome and Weyers acrodental dysostosis. Nat Genet 2000;24: 283-6

9. Galdzicka M, Patnala S, Hirshman MG, Cai JF, Nitowsky H; Egeland JA; Ginns EI. A new gene, EVC2, is mutated in Ellis-van Creveld syndrome. Molec Genet Metab 2002;77:291-5

10. Tompson SWJ; Ruiz-Perez VL, Blair HJ, et al. Sequencing EVC and EVC2 identifies mutations in two-thirds of Ellis-van Creveld syndrome patients. Hum Genet 2007;120: 663-70

11. Biggerstaff RH, Mazaheri M. Oral manifestations of the Ellis-van Creveld Syndrome. J Am Dent Assoc 1968;77:1090-5

12. Digoy PG, Greenberg M, Magit A. Congenital stridor secondary to an upper airway cyst in a patient with Ellis-van Creveld syndrome. Int J Pediatr Otorhinolaryngol 2005;69:1433-5
13. Giknis FL:Single atrium and the Ellis-van Creveld syndrome. J Pediatr 1963;62:558-64

14. Sajeev CG, Roy TNS, Venugopal K. Common atrium in a child with Ellis-Van Creveld syndrome. Heart 2002;88:142-2

15. Alvarez-Borja A. Ellis-van Creveld syndrome: report of two cases. Pediatrics 1960;26:301-9

16. Rosemberg S, Cameiro P C, Zerbini M C. Chondroectodermal dysplasia (Ellis-van Creveld) with anomalies of CNS and urinary tract. Am J Med Genet 1983;2:64-7

17. Zangwill KM, Boal DKB, Ladda RL. DandyWalker malformation in Ellis-van Creveld syndrome. Am J Med Genet 1988;31:123-9

18. Venkat-Raman N, Sebire NJ, Murphy KW, Carvalho JS, Hall CM. Increased firsttrimester fetal nuchal translucency thickness in association with chondroectodermal dysplasia (Ellis-Van Creveld syndrome). Ultrasound Obstet Gynecol. 2005;25:412-4

19. Guschmann M, Horn D, Gasiorek-Wiens A, Urban M, Kunze J, Vogel M. Ellis-van Creveld syndrome: examination at 15 weeks' gestation. Prenat Diagn 1999;19(9):879-83

20. 21. Horigome $H$, Hamada $H$, Sohda $S$, Oyake $Y$, Kurosaki Y.Prenatal ultrasonic diagnosis of a case of Ellis-van Creveld syndrome with a single atrium. Pediatr Radiol 1997;27(12): 942-4 